\title{
Prevalence and Correlates of Insomnia in a Polish Sample of Alcohol-Dependent Patients
}

\author{
Nataliya Zhabenko, Marcin Wojnar, and Kirk J. Brower
}

\begin{abstract}
Background: Insomnia is an important symptom in alcohol-dependent patients because it may persist despite abstinence and predispose to relapse to drinking. The goal of the present study was to evaluate the prevalence and clinical correlates of insomnia in a sample of 302 alcohol-dependent patients admitted to treatment programs in Poland.

Methods: Participants were mostly men (73.8\%) with a mean (SD) age of 43.5 (9.7) years. Insomnia in the past 1 month was assessed using a total score of 6 or higher on the Athens Insomnia Scale.

Results: Insomnia affected $62.9 \%$ of patients, and delayed sleep induction was the most common subtype. Insomnia was associated in bivariate analyses with less education, inadequate finances, problem drinking at an earlier age of onset, drinking frequency and quantity, drinking-related consequences, severity of alcohol and nicotine dependence, psychiatric and physical severity, and a childhood history of sexual or physical abuse $(p<0.05)$. Logistic regression analysis showed that mental and physical health status, severity of alcohol dependence, number of drinking days in the past 3 months, and childhood abuse were independent predictors of insomnia, explaining approximately 30 to $40 \%$ of the variance.

Conclusions: More than $60 \%$ of alcohol-dependent patients in a Polish sample screened positive for insomnia using a validated scale, a rate similar to those assessed with other scales in other countries. The study also showed that insomnia in alcohol-dependent patients is associated with poor physical health and childhood abuse, similar to the general population. The multifactorial nature of insomnia in alcohol-dependent patients has treatment implications.

Key Words: Athens Insomnia Scale, Alcohol Dependence, Childhood Abuse.
\end{abstract}

$I^{2}$ NSOMNIA IS A common problem among individuals with alcohol dependence (AD). In the general population, about $28 \%$ of respondents with past-year AD also had pastyear insomnia symptoms lasting 2 or more weeks compared to $13 \%$ overall (Crum et al., 2004). Because acute alcohol withdrawal typically lasts $<1$ week, it is unlikely that this increased rate is associated only with a withdrawal syndrome. Indeed, among alcohol-dependent individuals in the general population, the frequency of insomnia during acute alcohol withdrawal was nearly $50 \%$ (Brower and Perron, 2010a).

Among patients with $\mathrm{AD}$, insomnia symptoms occur in 36 to $91 \%$ of individuals, depending on sample characteristics as well as how and when insomnia was measured. Across 9

From the University of Michigan Addiction Research Center (NZ, KJB), Ann Arbor, Michigan; Department of Psychiatry (NZ), Luhansk State Medical University, Luhansk, Ukraine; and Medical Department of Psychiatry $(M W)$, University of Warsaw, Warsaw, Poland.

Received for publication August 5, 2011; accepted January 17, 2012.

Reprint requests: Kirk J. Brower, University of Michigan Addiction Research Center, 4250 Plymouth Road, SPC 5740, Ann Arbor, MI 48109-2700; Tel.: 734-232-0260; Fax: 734-998-7992; E-mail: kbrower@, umich.edu

Portions of this research were presented as a poster at the 34th Annual Scientific Meeting of the Research Society on Alcoholism, Atlanta, Georgia, June 25-29, 2011.

Copyright $(2012$ by the Research Society on Alcoholism.

DOI: 10.1111/j.1530-0277.2012.01771.x studies involving 2,133 patients, $56.2 \%$ of alcohol-dependent patients had insomnia symptoms (Baekeland et al., 1974; Bokström and Balldin, 1992; Brower et al., 2001; Caetano et al., 1998; Cohn et al., 2003; Escobar-Cordoba et al., 2009; Feuerlein, 1974; Foster et al., 2000; Mello and Mendelson, 1970).

In addition to their high frequency of occurrence, insomnia symptoms, especially difficulty falling asleep, are important because they have been associated with relapse to drinking (Brower et al., 2001; Foster and Peters, 1999; Skoloda et al., 1979). The correlates of insomnia in alcoholdependent patients, however, are not well-studied and findings are inconsistent. Demographic factors such as age and sex, in contrast to the general population and patients without AD, have not correlated with insomnia in most studies of alcohol-dependent patients (Baekeland et al., 1974; Brower et al., 2001), although women were more likely to have insomnia in a recent study (Brower et al., 2011). Four studies found correlations with depressive symptoms (Baekeland et al., 1974; Brower et al., 2001; Foster and Peters, 1999; Mackenzie et al., 1999), whereas 2 did not (Cohn et al., 2003; Escobar-Cordoba et al., 2009). Similarly, quantity or frequency of alcohol consumption has been reported to correlate with insomnia in some studies (Baekeland et al., 1974; Shinba et al., 1994) but not others (Brower et al., 2011; Cohn et al., 2003). Another area of inconsistency pertains to severity of AD. One indicator of dependence severity, a history of delirium tremens, was associated with 
insomnia in 1 study (Baekeland et al., 1974), but not another (Feuerlein, 1974). Other indicators of dependence severity, such as scores on the Michigan Alcoholism Screening Test (MAST) and Diagnostic and Statistical Manual of Mental Disorders, Fourth Edition (DSM-IV) symptom count correlated significantly with insomnia in 1 study (Brower et al., 2001), whereas scores on the Severity of AD Questionnaire were not significantly correlated with insomnia in another study (Foster and Peters, 1999). Of note, Brower and colleagues (2011) found a bivariate correlation between insomnia and DSM-IV symptom count that did not remain significant when other variables were analyzed concurrently. Thus, some inconsistencies in the literature may stem from the presence or absence of covariate analyses.

Interestingly, none of these studies included physical health status, cigarette smoking, or childhood abuse, yet a relationship between physical health and insomnia is well-established in the general population (Doi et al., 2003; Janson et al., 2001; Pallesen et al., 2001), as is a relationship between cigarette smoking and insomnia (Peters et al., 2011; Pomerleau et al., 2000; Soldatos et al., 1980; Wetter and Young, 1994). Moreover, cigarette users who smoke during nighttime awakenings report significantly greater sleep disturbance than those who do not smoke during the night (Peters et al., 2011), and other studies have shown that insomnia predicts relapse among smokers trying to quit (Brower and Perron, 2010b). Finally, 5 studies investigated sleep disorders among women with a history of childhood sexual abuse (Briere and Runtz, 1987; McCauley et al., 1997; Noll et al., 2006; Pigeon et al., 2009; Teegen, 1999). Rates of sleep disturbance in women with a childhood history of sexual abuse are higher (Noll et al., 2006) and more severe (Briere and Runtz, 1987) than in women without such a history. These rates range from 43 to 88\% (Briere and Runtz, 1987; McCauley et al., 1997; Pigeon et al., 2009; Teegen, 1999), and are not limited to nightmares. Only 2 of these studies, however, also examined childhood physical abuse (Briere and Runtz, 1989; McCauley et al., 1997) and none investigated sleep among male abuse victims.

Therefore, the goal of the present study was to evaluate the prevalence and clinical correlates of insomnia in a sample of alcohol-dependent patients admitted to treatment programs in Poland, including some correlates in the general population that have not been previously studied in alcohol-dependent patients. A secondary goal was to investigate which subtypes of insomnia are most commonly reported in terms of delayed sleep induction (sleep-onset insomnia), awakenings during the night, and final awakening earlier than desired. Of these subtypes, sleep-onset insomnia has been most consistently associated with relapse to drinking (Brower et al., 1998, 2001; Conroy et al., 2006; Foster and Peters, 1999; Skoloda et al., 1979). We hypothesized that mental and physical health severity, nicotine dependence, and childhood abuse would independently predict insomnia, whereas alcohol consumption and dependence severity would have bivariate correlations with insomnia, but not remain in a model with other significant correlates of insomnia.

\section{MATERIALS AND METHODS}

\section{Participants}

Participants were 304 patients recruited from outpatient and inpatient abstinence-based, alcohol treatment programs in Warsaw, Poland. Inpatient programs lasted for no longer than 8 weeks, and treatment included intensive daily group therapy and 3 hours a week of individual therapy. Outpatient programs combined group and individual therapy for 3 days a week at 3 hours per day, and lasted for 12 to 26 weeks. All programs predominantly used a cognitive-behavioral approach to therapy. Pharmacotherapy was not utilized.

Only patients with a DSM-IV-TR diagnosis of AD were eligible to participate (American Psychiatric Association, 2000). Diagnoses were made by the consensus of a multidisciplinary team specializing in the treatment of addictions that included a psychiatrist and an addictions therapist. Patients were excluded if they had a psychotic disorder or required medication for a psychiatric disorder. All subjects signed written informed consent to participate in the study, which was approved by both the University of Michigan Institutional Review Board and the Bioethics Committee at the Medical University of Warsaw.

Two patients were excluded because they had missing data in the sleep questionnaire. Of the remaining 302 patients, $268(88.7 \%)$ were recruited from inpatient treatment programs. The 2 treatment groups did not significantly differ in terms of insomnia, so they were combined for analyses. Participants were European Caucasians, had a mean (SD) age of $43.5(9.7)$ years, and $73.8 \%$ were men. The majority of the patients were unemployed (61.5\%), half $(50.3 \%)$ were married, and their mean years of education was 12.2 (2.6). Additional descriptive characteristics of the sample are provided in Table 1.

\section{Measures}

The Athens Insomnia Scale (AIS) is an 8-item self-administered questionnaire, which was used to assess insomnia symptoms during the 1 month prior to treatment entry. The items assess sleep induction time (time it takes to fall asleep or sleep-onset insomnia); awakenings during the night; final awakening earlier than desired; total sleep duration; overall quality of sleep; and daytime impairment (sense of well-being during the day, functioning during the day, and sleepiness during the day), with item scores ranging from 0 (no problem) to 3 (serious problem) and total scores ranging from 0 to 24. An AIS score of 6 or higher defines a positive screen for insomnia (Soldatos et al., 2000, 2005).

The University of Arkansas Substance Abuse Outcomes Module is a self-report tool that collects information about demographics, substance use, and social functioning (Smith et al., 2006). In addition, patients were asked whether or not they had any kind of sexual contact against their wishes before age 18 (childhood sexual abuse), and whether before age 18 they were hit, beaten, or physically abused so much that they feared for their safety or had marks (childhood physical abuse).

The Fagerstrom Test for Nicotine Dependence (FTND) was used to identify severity of nicotine dependence. It is a highly reliable, self-administered 6-item questionnaire, with a total score ranging between 0 and 10, and higher scores indicating more dependence (Heatherton et al., 1991). For this study, nonsmokers were scored with a zero on the FTND and classified as nicotine nondependent.

The Time-Line Follow-Back interview was used to assess the frequency and quantity of alcohol intake in the 3 months prior to the assessment, as well as days since last drink. It is a reliable, valid, interviewer-administered calendar method to evaluate daily patterns and frequency of alcohol consumption (Sobell and Sobell, 1992).

The Polish version (Falicki et al., 1986; Habrat, 1988) of the 25-item MAST was used to measure the lifetime severity of AD. 
Table 1. Bivariate Comparisons Between Patients With and Without Insomnia

\begin{tabular}{|c|c|c|c|c|}
\hline Characteristics & Total sample $(n=302)$ & Insomnia $(n=190)$ & No insomnia $(n=112)$ & $p$-Value \\
\hline \multicolumn{5}{|l|}{ Demographic variables } \\
\hline Age (years) ${ }^{a}$ & $43.5 \pm 9.7$ & $43.6 \pm 9.1$ & $43.3 \pm 10.6$ & 0.823 \\
\hline Gender (men) ${ }^{\mathrm{b}}$ & $223(73.8)$ & $141(74.2)$ & $82(73.2)$ & 0.956 \\
\hline Education (years) ${ }^{c}$ & $12(11-13)$ & $11(11-13)$ & $12(11-14)$ & 0.004 \\
\hline Employed & $116(38.5)$ & $67(35.4)$ & $49(43.8)$ & 0.191 \\
\hline Married & $152(50.3)$ & 93 (48.9) & $59(52.7)$ & 0.612 \\
\hline Enough money for needs & $104(34.8)$ & 53 (28.2) & $51(45.9)$ & 0.003 \\
\hline \multicolumn{5}{|l|}{ Alcohol-related variables } \\
\hline Age at onset of drinking problems (years) & $22(18-29)$ & $20(18-28)$ & $22(19-31)$ & 0.035 \\
\hline No. of drinking days in past 90 days & $26(4-52)$ & $33(6-57)$ & $20(0-36)$ & 0.006 \\
\hline SIP score & $24.1 \pm 11.1$ & $26.3 \pm 10.1$ & $20.4 \pm 11.6$ & 0.000 \\
\hline No. of drinks in past 90 days & $582.5(267.5-968)$ & $657(288-1,146)$ & $502.5(262-725)$ & 0.008 \\
\hline No. of days since last drink & $40.0(25.0-67.0)$ & $37.0(20.0-57.8)$ & $48.5(33.0-77.5)$ & 0.019 \\
\hline MAST score & $34.6 \pm 9.6$ & $36.2 \pm 9.1$ & $31.8 \pm 9.8$ & 0.000 \\
\hline \multicolumn{5}{|l|}{ Comorbidity factors } \\
\hline MCS score & $38.3 \pm 11.9$ & $34.6 \pm 11.1$ & $44.4 \pm 10.7$ & 0.000 \\
\hline PCS score & $46.7 \pm 9.9$ & $44.5 \pm 9.4$ & $50.2 \pm 9.8$ & 0.000 \\
\hline Current smoker & $242(80.1)$ & $151(79.5)$ & $91(81.3)$ & 0.822 \\
\hline FTND total score & $6(2-7)$ & $6(3-8)$ & $5(2-7)$ & 0.048 \\
\hline Used other drugs (past 28 days) & $43(14.2)$ & $26(13.7)$ & $17(15.2)$ & 0.850 \\
\hline Childhood abuse history & $98(34.4)$ & 75 (39.5) & $23(20.5)$ & 0.001 \\
\hline
\end{tabular}

${ }^{a}$ Normally distributed continuous variables expressed as mean \pm standard deviation.

${ }^{b}$ Dichotomous variables expressed as frequency (\%).

${ }^{\mathrm{C}}$ Nonnormally distributed variables expressed as median (interquartile range).

${ }^{*}$ Chi-square, $t$-test, or Mann-Whitney U-test was performed where appropriate. Significant results are bolded.

SIP, Short Inventory of Problems; MAST, Michigan Alcoholism Screening Test; MCS, mental component summary; PCS, physical component summary; FTND, Fagerstrom Test for Nicotine Dependence scale.

The MAST is self-administered with questions about the effects of alcohol on respondents' interpersonal relationships, work performance, and health (Gibbs, 1983; Selzer, 1971).

The 15-item Short Inventory of Problems (SIP) was used to assess the level of drinking-related consequences in the month prior to treatment admission. It is a short version of the Drinker Inventory of Consequences, which was developed for Project MATCH to evaluate adverse consequences of drinking (Forcehimes et al., 2007).

The 36-item short-form (SF-36) General Health Survey was referenced to the 4 weeks prior to treatment entry, and includes 2 component summary scores representing physical and mental health status (the PCS and MCS, respectively). Both the PCS and MCS have a mean of 50 and a standard deviation of 10 in the general population. Lower scores reflect a lower state of health, more functional limitation and distress, and greater social and role disability (Ware and Kosinski, 2001; Ware and Sherbourne, 1992). The SF-36 has been used in several studies to measure the level of impairment associated with sleep problems (Leger and Bayon, 2010). It has also been used in several Polish studies to evaluate patients with depressive symptoms, gastrointestinal problems, and cardiovascular diseases (Dudek et al., 2007; Regula et al., 2005; Szczesniak et al., 2008).

\section{Statistical Analysis}

All statistical techniques were performed in SPSS, version 18 (IBM, Armonk, NY). A categorical variable of insomnia was created, using an AIS score of 6 or higher to define insomnia. Continuous variables were tested for normality. Descriptive statistics for normally distributed variables are presented as means and standard deviations, whereas medians and interquartile ranges (IQRs) are presented for nonnormal variables. Bivariate analyses examined associations between baseline variables and insomnia. Chi-square tests were used to analyze the relationship between categorical variables. The $t$-test for independent samples and the nonparametric alternative Mann-Whitney $U$-test (when appropriate) were used to compare patients with and without insomnia on continuous measures. Those variables that were significantly $(p<0.05)$ associated with insomnia in the bivariate analyses were entered into a logistic regression analysis. To model the best predictors of insomnia we employed the backward method of binary logistic regression (Tabachnick and Fidell, 2001). This method is used for exploratory work, not constrained by a priori hypotheses. Although some prior work on correlates of insomnia in AD has been published as reviewed above, this is the first study in a Polish population and with the AIS; so we let the present data guide the model as a basis to generate hypotheses for future work in this area.

\section{RESULTS}

\section{Prevalence and Subtypes of Insomnia in the Study Sample}

Insomnia affected $190(62.9 \%)$ of 302 patients. Of those, $62.1 \%$ had delayed sleep induction (sleep-onset insomnia), $56.9 \%$ endorsed awakenings during the night, and $41.0 \%$ reported final awakening earlier than desired (Table 2). Frequencies of other AIS symptoms among those with and without insomnia are shown in Table 2. A minority of patients who did not meet criteria for insomnia (AIS score $<6$ ) nonetheless reported moderate or severe symptoms of insomnia, as indicated by item scores of 2 or 3 . Specifically, $6.3 \%$ reported considerable or serious problems with awakening during the night, 5.4\% reported markedly delayed sleep induction, and $4.5 \%$ experienced sleepiness during the day (Table 2). 
Table 2. Types of Insomnia and Item Scores in a Sample of AlcoholDependent Patients (Athens Insomnia Scale)

\begin{tabular}{|c|c|c|c|}
\hline & $0^{*}$ & $1^{*}$ & 2 and $3^{*}$ \\
\hline \multicolumn{4}{|c|}{ Delayed sleep induction; $n(\%)$} \\
\hline Insomniacs & $12(6.3)$ & $60(31.6)$ & $118(62.1)$ \\
\hline Noninsomniacs & $62(55.4)$ & 44 (39.3) & $6(5.4)$ \\
\hline \multicolumn{4}{|c|}{ Awakenings during the night; $n(\%)$} \\
\hline Insomniacs & $5(2.6)$ & $77(40.5)$ & $108(56.9)$ \\
\hline Noninsomniacs & $32(28.6)$ & $73(65.2)$ & $7(6.3)$ \\
\hline \multicolumn{4}{|c|}{ Final awakening earlier than desired; $n(\%)$} \\
\hline Insomniacs & $29(15.3)$ & $83(43.7)$ & $78(41.0)$ \\
\hline Noninsomniacs & $60(53.6)$ & $50(44.6)$ & $2(1.8)$ \\
\hline \multicolumn{4}{|c|}{ Total sleep duration; $n(\%)$} \\
\hline Insomniacs & $27(14.2)$ & $83(43.7)$ & $80(42.1)$ \\
\hline Noninsomniacs & 91 (81.3) & $21(18.8)$ & - \\
\hline \multicolumn{4}{|c|}{ Overall quality of sleep; $n(\%)$} \\
\hline Insomniacs & $13(6.8)$ & $102(53.7)$ & $75(39.5)$ \\
\hline Noninsomniacs & $86(76.8)$ & $26(23.2)$ & - \\
\hline \multicolumn{4}{|c|}{ Sense of well-being during the day; $n(\%)$} \\
\hline Insomniacs & $27(14.2)$ & $102(53.7)$ & $61(32.1)$ \\
\hline Noninsomniacs & $102(91.1)$ & $10(8.9)$ & - \\
\hline \multicolumn{4}{|c|}{ Functioning (physical and mental) during the day; $n(\%)$} \\
\hline Insomniacs & $28(14.7)$ & $116(61.1)$ & $46(24.2)$ \\
\hline Noninsomniacs & $93(83.8)$ & $18(16.2)$ & - \\
\hline \multicolumn{4}{|c|}{ Sleepiness during the day; $n(\%)$} \\
\hline Insomniacs & $12(6.3)$ & $107(56.3)$ & $71(37.4)$ \\
\hline Noninsomniacs & $26(23.4)$ & 80 (72.1) & $5(4.5)$ \\
\hline
\end{tabular}

*0 corresponds to "no problem at all"; 1 corresponds to "minor problem"; 2 and 3 correspond to "considerable and serious problem," respectively.

\section{Bivariate Comparisons Between Patients With and Without Insomnia}

No significant differences between patients with and without insomnia were found for age, gender, marital status, or employment (Table 1). Patients with insomnia were significantly less satisfied with their financial state (28.2 vs. $45.9 \%$, $p<0.05$ ), and had significantly less education (median 11 [IQR: 11 to 13] years) than those without insomnia (median 12 [IQR: 11 to 14] years). The 2 groups did not differ significantly in terms of other substances use, including tobacco smoking or not; however, patients with insomnia reported greater nicotine dependence $(p<0.05)$. Altogether, $14.2 \%$ of patients admitted using 1 or more drugs other than tobacco in the previous 28 days, including sedative-hypnotics $(9.3 \%)$, cannabis $(4.6 \%)$, cocaine $(1.0 \%)$, other stimulants $(3.3 \%)$, and opioids $(1.0 \%)$. Patients with insomnia also used alcohol more frequently in the past 90 days $(p<0.05)$ and consumed a greater number of drinks over the last 3 months $(p<0.05)$, and they were abstinent for a shorter period of time prior to the assessment $(p<0.05)$. Patients with insomnia had an earlier age of onset for alcohol problems. Patients with insomnia also had significantly higher MAST scores as well as higher SIP scores, indicating a greater severity of both dependence and alcohol-related problems (physical, intrapersonal, social responsibility, interpersonal, and impulse control). As predicted, patients with insomnia had significantly lower MCS and PCS scores, indicating worse health status, than patients without insomnia. Finally, patients with insomnia were significantly more likely than those without insomnia to report a childhood history of either sexual or physical abuse (39.5 vs. $20.5 \%, p=0.001)$.

\section{Multivariate Analysis of Insomnia Predictors}

All significant variables from the bivariate analyses were included in the logistic regression analysis except for quantity of alcohol consumption in the prior 90 days, because it was strongly correlated with frequency of alcohol consumption (Spearman $r=0.84$ ) and the latter variable had fewer missing data values. Similarly, days since last drink was excluded, because it also correlated strongly with frequency of alcohol consumption (Spearman $r=0.61$ ) and had more missing values than frequency of alcohol consumption. The model was statistically significant and retained 5 of the original 10 variables: mental and physical health (each $p<0.0005$ ), frequency of drinking days over the last 90 days $(p=0.008)$, childhood abuse history $(p=0.010)$, and severity of AD $(p=0.025)$. Missing data reduced the sample size to 241 $(79.8 \%)$ of 302 patients. Therefore, a second logistic regression analysis was performed, entering only the 5 variables that were significant in the exploratory model and forcing them to stay in the model. This increased the sample size to $287(95.0 \%)$ participants, and confirmed that each of the 5 variables remained significant, independent predictors (Table 3). Overall, the model was statistically significant $\left(\chi^{2}=99.1, \mathrm{df}=5, p<0.0005\right)$ and explained between $29.2 \%$ $\left(\right.$ Cox and Snell $R^{2}$ ) and 39.7\% (Nagelkerke $R^{2}$ ) of the variance in insomnia. The strongest correlates of insomnia were mental and physical health status. For each 5-point worsening in mental and physical health scores, respectively, the odds of insomnia increased by $40 \%$. Similarly, the odds of insomnia increased by $18 \%$ for each additional 10 days of drinking, and patients with greater severity of $\mathrm{AD}$ were more likely to report insomnia $(\mathrm{OR}=1.045)$. Finally, patients who reported childhood abuse were 2.4 times more likely to report insomnia than those who did not.

Table 3. Binary Logistic Regression Analysis for Predictors of Insomnia

\begin{tabular}{lcccc}
\hline Predictor variables & $B$ & OR & $95 \% \mathrm{Cl}$ & $p$-Value \\
\hline $\begin{array}{l}\text { Mental component summary } \\
\quad \text { (MCS) score }\end{array}$ & -0.078 & 0.925 & $0.900-0.951$ & 0.000 \\
$\begin{array}{l}\text { Physical component summary } \\
\quad \text { (PCS) score }\end{array}$ & -0.074 & 0.929 & $0.900-0.958$ & 0.000 \\
$\begin{array}{l}\text { Number of drinking days in last } \\
\quad 90 \text { days }\end{array}$ & 0.018 & 1.018 & $1.006-1.030$ & 0.002 \\
$\begin{array}{l}\text { MAST (total score) } \\
\text { Childhood abuse history }\end{array}$ & 0.044 & 1.045 & $1.012-1.080$ & 0.007 \\
& 0.866 & 2.378 & $1.227-4.610$ & 0.010
\end{tabular}

Final model $(n=287)$. Initially 10 variables were explored with the backward method of binary logistic regression (satisfaction with financial state, education, age at onset of alcohol problems, number of drinking days over the last 90 days, Short Inventory of Problems score, Michigan Alcoholism Screening Test (MAST) score, nicotine dependence severity, MCS, PCS, and childhood abuse). Only significant variables (MCS, PCS, MAST score, number of drinking days over the last 90 days, childhood abuse) from the first model were entered into the final model (enter method). 


\section{DISCUSSION}

The aims of the study were to evaluate the prevalence and subtypes of insomnia in alcohol-dependent patients and to assess associations between insomnia and other patient characteristics. The rate of insomnia as defined by the AIS in this study $(62.9 \%)$ is consistent with previous studies of insomnia in alcohol-dependent patients, which range from 36 to $91 \%$. By comparison, an international study of 35,327 individuals in the general population from 10 countries (not including Poland) utilized and validated the AIS, and found a global rate of insomnia of $31.6 \%$ (Soldatos et al., 2005).

Our results also point to the importance of sleep-onset insomnia in patients with AD. The results are consistent with Currie and colleagues (2003) who found that problems getting to sleep were worse than sleep maintenance difficulties in recovering alcohol-dependent patients. Moreover, difficulty falling asleep is significantly associated with subsequent relapse in alcohol-dependent patients (Brower, 2003). Thus, it may be important to assess the subtype of insomnia, and scales such as the AIS can assist the clinician.

The independent correlates of insomnia in this study were physical health, mental health, frequency of recent drinking, severity of $\mathrm{AD}$, and childhood abuse history. The associations between insomnia and both physical and mental health were expected, because they are also characteristic of the general population (Taylor et al., 2003), although physical health has not been included in previous studies of insomnia correlates in alcohol-dependent patients. In contrast to the general population, however, alcohol-dependent patients are more likely to suffer pervasive physical health difficulties (Clark et al., 2001; David et al., 2004; Glenn et al., 1989). In this study, the PCS score of 44.5 in alcohol-dependent patients with insomnia is very similar to the score of 43.1, which was reported in 103 patients with AD presenting as primary care outpatients (Volk et al., 1997), but markedly higher (better) than the score of 34.4 among alcoholdependent patients who visited a Veterans Administration (VA) facility for any symptom or condition during the previous year (Kalman et al., 2004). By contrast, the MCS score of 34.6 for alcohol-dependent patients with insomnia was lower (worse) than the corresponding scores, 40.5 and 42.2, for patients with AD from primary care (Volk et al., 1997) and the VA (Kalman et al., 2004), respectively. However, VA patients with co-occurring AD and other psychiatric disorders had a mean MCS score of 33.3 (Kalman et al., 2004), which is equivalent to our finding of 34.6.

Like the MCS which measures general mental health, a recent study of alcohol-dependent individuals in the United States that utilized the Brief Symptom Inventory (BSI; Derogatis and Melisaratos, 1983) to assess overall psychiatric distress also found a significant relationship with insomnia (Brower et al., 2011). Other studies of alcohol-dependent patients have found an association between depressive symptoms and insomnia (Currie et al., 2003; Foster and Peters,
1999), but not always (Cohn et al., 2003; Escobar-Cordoba et al., 2009). It may be that depression-specific measures do not detect anxiety and other stress-induced mental states that contribute to insomnia, in contrast to measures such as the MCS and BSI. Clinicians should look more broadly than depressive states when assessing psychological factors affecting insomnia.

The association of insomnia with drinking frequency is consistent with some (Baekeland et al., 1974; Shinba et al., 1994) but not all (Brower et al., 2011; Foster and Peters, 1999) studies correlating consumption with insomnia in alcohol-dependent patients. Nevertheless, many alcohol-dependent individuals consume alcohol to aid their sleep, yet alcohol consumption before bedtime is known to disrupt sleep. Some (Baekeland et al., 1974; Brower et al., 2011) but not all (Cohn et al., 2003; Foster and Peters, 1999) studies have also found a relationship between severity of $\mathrm{AD}$ and insomnia. We assumed incorrectly that severity of AD would not predict insomnia after controlling for other variables, based on these inconsistent results and our own previous study that found only a bivariate relationship (Brower et al., 2011). That both mental health severity and alcohol-specific variables were independent predictors has clear treatment implications in that both should be addressed to improve sleep.

A history of childhood abuse was associated with more than a 2-fold increase in odds for insomnia, while controlling for mental health status, indicating that childhood trauma can influence insomnia as an adult through a pathway separate from mental health problems. Given that nightmares are not assessed by the AIS, it can be assumed that sleep was more broadly affected in this sample. This is the first study to our knowledge, however, that reports this finding in alcoholdependent patients, and the first to assess that relationship in a predominantly male sample.

Of particular interest is the independent contribution of childhood abuse to adult insomnia in this study. Other longterm effects of child abuse reported in the literature include self-destructive behavior, anxiety, depression, poor selfesteem, difficulty trusting others, anger and hostility, and substance use disorders (Beitchman et al., 1992; Langeland and Hartgers, 1998). Yet the contribution of childhood abuse to adult insomnia was independent of mental health and substance use variables in this study. Without data on order of onset and duration of insomnia, we can only, but reasonably, speculate that insomnia may be an early and persistent response to childhood abuse, occurring prior to the age at which heavy drinking habits and AD develop, and obviously prior to mental health functioning as an adult. In other words, the independent effect of childhood abuse may possibly be explained by an association with insomnia that is early in onset and primary in relation to both $\mathrm{AD}$ and adult mental health functioning. In this regard, it is noteworthy that childhood sleep problems have been shown in longitudinal research to be a predictor of early onset drinking and substance-related problems during adolescence and young adulthood (Wong et al., 2004, 2010). Moreover, cross- 
sectional research indicates that more than $50 \%$ of alcoholdependent patients reported that insomnia preceded onset of their AD (Currie et al., 2003).

The majority of studies in the general population have shown a significant association between smoking status and insomnia (Peters et al., 2011; Pomerleau et al., 2000; Soldatos et al., 1980; Wetter and Young, 1994). Although smoking status per se was not associated with insomnia in this study, the severity of nicotine dependence was significantly related, but only before and not after controlling for other variables. Interestingly, average FTND scores differed by only 1 point in patients with and without insomnia (medians of 6 and 5, respectively), yet Fagerstrom and colleagues (1990), suggested that difference be used as the cutoff between high and medium nicotine dependence.

A number of demographic variables such as age, sex, marital status, and employment have been associated with insomnia in some general population studies (Ford and Kamerow, 1989; Zhang and Wing, 2006); however, the negative results here parallel other studies with alcohol-dependent patients, which found no relationships with age (Baekeland et al., 1974; Brower et al., 2001, 2011), sex (Brower et al., 2001; Escobar-Cordoba et al., 2009), and marital status (Brower et al., 2001, 2011). Another study of AD found employment was associated with insomnia, but not after controlling for other variables (Brower et al., 2011). Nevertheless, Brower and colleagues (2011) found that women with AD were more likely than alcohol-dependent men to have insomnia while controlling for other variables, but that sample consisted of outpatients and individuals from the community not in treatment. Like this study, the other 2 negative studies for an effect of sex included mostly inpatients (Brower et al., 2001; EscobarCordoba et al., 2009), a status that is likely associated with greater illness severity, and hence insomnia, regardless of sex. Lower education has also been associated with insomnia in the general population (Gellis et al., 2005; Li et al., 2002), but not in other studies of alcohol-dependent patients (Brower et al., 2001, 2011). In this study, education was significant in the bivariate analysis, but not in the logistic model.

Some limitations of this study should be noted. First, the AIS is a well-validated screening tool, but no diagnostic confirmation of insomnia via a clinical interview occurred in this study, nor were any objective measures of sleep included. Second, the sample size was relatively strong for statistical purposes, but the Polish patients studied here were racially and ethnically homogeneous and recruited from treatment sites, so the results may not extend to other groups of alcohol-dependent individuals. Third, we did not assess if the onset of insomnia preceded the onset of drinking problems, which can affect the persistence and independence of insomnia symptoms, apart from drinking variables. Fourth, being a childhood abuse survivor is an important problem with serious long-term sequelae (Beitchman et al., 1992; KendallTackett, 2002) that might lead to premorbid insomnia as well, yet information about childhood abuse was limited in this study to a categorical yes-no variable. Therefore, the potential effects of the duration, onset, and other circumstances of abuse on insomnia and its onset are not known, which may be important (Bagley and Ramsay, 1986; Beitchman et al., 1992; Browne and Finkelhor, 1995). Including such variables in future studies of alcohol-dependent patients may lead to a better understanding of the relationships between them and insomnia. In regard to trauma history, nightmares are also important to assess, which are not covered in the AIS or this study. Fifth, comorbid psychiatric diagnoses were not included in the main analyses, because only a subset of 141 patients was assessed by a structured diagnostic interview, the Mini-International Neuropsychiatric Interview (Sheehan et al., 1998). While psychiatric disorders requiring medication was an exclusion criterion for the study, $44(31.2 \%)$ of 141 patients had either a current mood or anxiety disorder, including $24.6 \%$ with a mood disorder (major depression, dysthymic disorder, or hypomania) and $12.1 \%$ with an anxiety disorder (generalized anxiety disorder, panic disorder, posttraumatic stress disorder, or obsessive-compulsive disorder). Those with a comorbid psychiatric disorder were significantly more likely to have insomnia than those without a co-occurring disorder $(70.5$ vs. $\left.48.5 \%, \chi^{2}=5.93, \mathrm{df}=1, p=0.015\right)$. When both the presence/absence of a comorbid psychiatric disorder and mental health scores were entered into a logistic regression analysis with insomnia as the dependent variable, however, only mental health scores remained significant ( $p=0.002$ vs. 0.268 ). Therefore, mental health scores likely served as a reasonable proxy for psychiatric diagnosis in this study.

In conclusion, this study evaluated the frequency and correlates of insomnia in a sample of alcohol-dependent Polish patients. More than $60 \%$ of patients screened positive for insomnia using the AIS, which confirms the importance of using such scales in clinical settings. This study also clearly demonstrated the multifactorial nature of insomnia that is associated with $\mathrm{AD}$, including the importance of physical health status and childhood abuse history, in addition to previously reported correlates such as mental health status, alcohol consumption, and severity of AD. The present findings highlighted that these were independent predictors of insomnia among alcohol-dependent patients. The multifactorial nature and independence of predictors may help to explain why insomnia persists despite abstinence in some patients, while simultaneously underlining the contribution and importance of addressing alcohol-related factors in treatment. Inquiring about childhood abuse history is especially important because of its role in insomnia and other health outcomes. Although replication and extension of the current findings is warranted, the study results can inform clinical practice about factors to assess, treat, and monitor when responding to complaints of insomnia among alcohol-dependent patients.

\section{ACKNOWLEDGMENTS}

KJB and MW designed the study and wrote the protocol. $\mathrm{NZ}$ conducted literature searches and statistical analysis. 
KJB and NZ wrote the first draft of the manuscript. All authors contributed to and have approved the final manuscript. This work was supported by the Fogarty International Center/NIDA International Substance Abuse Research Program grant D43 TW005818, NIAAA grants R21 AA016104 and K24 AA00304, and the Polish Ministry of Science and Higher Education Grant 2P05D 00429.

\section{REFERENCES}

American Psychiatric Association (2000) Diagnostic and Statistical Manual of Mental Disorders: DSM-IV-TR. American Psychiatric Pub. Inc., Washington, DC.

Baekeland F, Lundwall L, Shanahan T, Kissin B (1974) Clinical correlates of reported sleep disturbance in alcoholics. Q J Stud Alcohol 35:1230-1241.

Bagley C, Ramsay R (1986) Sexual abuse in childhood. J Soc Work Hum Sex 4:33-47.

Beitchman KJ, Joseph H, Cassavia E (1992) A review of the long-term effects of child sexual abuse. Child Abuse Negl 16:101-118.

Bokström K, Balldin J (1992) A rating scale for assessment of alcohol withdrawal psychopathology (AWIP). Alcohol Clin Exp Res 16:241-249.

Briere J, Runtz M (1987) Post sexual abuse trauma. J Interpersonal Viol 2:367-379.

Briere J, Runtz M (1989) The trauma symptom checklist (TSC-33). J Interpersonal Viol 4:151.

Brower KJ (2003) Insomnia, alcoholism and relapse. Sleep Med Rev 7: 523-539.

Brower KJ, Aldrich MS, Hall JM (1998) Polysomnographic and subjective sleep predictors of alcoholic relapse. Alcohol Clin Exp Res 22:18641871.

Brower KJ, Aldrich MS, Robinson EA, Zucker RA, Greden JF (2001) Insomnia, self-medication, and relapse to alcoholism. Am J Psychiatry 158:399-404.

Brower KJ, Krentzman A, Robinson EAR (2011) Persistent insomnia, abstinence, and moderate drinking in alcohol-dependent individuals. Am J Addict 20:435-440.

Brower KJ, Perron BE (2010a) Prevalence and correlates of withdrawalrelated insomnia among adults with alcohol dependence: results from a national survey. Am J Addict 19:238-244.

Brower KJ, Perron BE (2010b) Sleep disturbance as a universal risk factor for relapse in addictions to psychoactive substances. Med Hypotheses 74:928-933.

Browne A, Finkelhor D (1995) Impact of child sexual abuse: a review of the research. in Short- and Long-Term Effects (Child Abuse: A Multidisciplinary Survey) (Finkelman BP ed), pp 60-72. Garland Publishing, New York, NY \& London.

Caetano R, Clark C, Greenfield T (1998) Prevalence, trends, and incidence of alcohol withdrawal symptoms. Alcohol Health Res World 22:73-79.

Clark DB, Lynch KG, Donovan JE, Block GD (2001) Health problems in adolescents with alcohol use disorders: self report, liver injury, and physical examination findings and correlates. Alcohol Clin Exp Res 25:1350-1359.

Cohn TJ, Foster JH, Peters TJ (2003) Sequential studies of sleep disturbance and quality of life in abstaining alcoholics. Addict Biol 8:455-462.

Conroy DA, Todd Arnedt J, Brower KJ, Strobbe S, Consens F, Hoffmann R, Armitage R (2006) Perception of sleep in recovering alcohol-dependent patients with insomnia: relationship with future drinking. Alcohol Clin Exp Res 30:1992-1999.

Crum RM, Ford DE, Storr CL, Chan YF (2004) Association of sleep disturbance with chronicity and remission of alcohol dependence: data from a population based prospective study. Alcohol Clin Exp Res 28:1533-1540.

Currie SR, Clark S, Rimac S, Malhotra S (2003) Comprehensive assessment of insomnia in recovering alcoholics using daily sleep diaries and ambulatory monitoring. Alcohol Clin Exp Res 27:1262-1269.
David D, Woodward C, Esquenazi J, Mellman TA (2004) Comparison of comorbid physical illnesses among veterans with PTSD and veterans with alcohol dependence. Psych Serv 55:82-85.

Derogatis LR, Melisaratos N (1983) The brief symptom inventory: an introductory report. Psychol Med 13:595-605.

Doi Y, Minowa M, Tango T (2003) Impact and correlates of poor sleep quality in Japanese white-collar employees. Sleep 26:467-471.

Dudek D, Datka W, Siwek M, Wrobel A, Zieba A (2007) The quality of life related to depressive symptoms in coronary artery disease patients after successful coronary angioplasty: one-year follow-up. Polish Psych 41: 229-242.

Escobar-Cordoba F, Avila-Cadavid JD, Cote-Menendez M (2009) Complaints of insomnia in hospitalized alcoholics. Rev Bras Psiquiatr 31:261-264.

Fagerstrom K, Heatherton T, Kozlowski L (1990) Nicotine addiction and its assessment. Ear Nose Throat J 69:763-775.

Falicki Z, Karczewski J, Wandzel L, Chrzanowski W (1986) Usefulness of the Michigan alcoholism screening test (MAST) in Poland. Psychiatr Pol 20:38-42.

Feuerlein W (1974) The acute alcohol withdrawal syndrome: findings and problems. Br J Addict Alcohol Other Drugs 69:141-148.

Forcehimes A, Tonigan J, Miller W, Kenna G, Baer J (2007) Psychometrics of the drinker inventory of consequences (DrInC). Addict Behav 32:16991704.

Ford D, Kamerow D (1989) Epidemiologic study of sleep disturbances and psychiatric disorders: an opportunity for prevention? JAMA 262:14791484.

Foster J, Marshall E, Peters T (2000) Application of a quality of life measure, the life situation survey (LSS), to alcohol dependent subjects in relapse and remission. Alcohol Clin Exp Res 24:1687-1692.

Foster J, Peters T (1999) Impaired sleep in alcohol misusers and dependent alcoholics and the impact upon outcome. Alcohol Clin Exp Res 23:1044 1051.

Gellis L, Lichstein K, Scarinci I, Durrence H, Taylor D, Bush A, Riedel B (2005) Socioeconomic status and insomnia. J Abnorm Psychol 114:111118.

Gibbs L (1983) Validity and reliability of the Michigan alcoholism screening test: a review. Drug Alcohol Depend 12:279-285.

Glenn SW, Parsons OA, Stevens L (1989) Effects of alcohol abuse and familial alcoholism on physical health in men and women. Health Psychol 8: 325-341.

Habrat B (1988) Questionnaire methods in the diagnosis and evaluation of alcohol dependence. Psychiatr Pol 22:149-155.

Heatherton TF, Kozlowski LT, Frecker RC (1991) The Fagerstrom test for nicotine dependence: a revision of the Fagerstrom tolerance questionnaire. Br J Addict 86:1119-1127.

Janson C, Lindberg E, Gislason T, Elmasry A, Boman G (2001) Insomnia in men: a 10-year prospective population based study. Sleep 24:425-430.

Kalman D, Lee A, Chan E, Miller DR, Spiro A III, Ren XS, Kazis LE (2004) Alcohol dependence, other psychiatric disorders, and health-related quality of life: a replication study in a large random sample of enrollees in the Veterans Health Administration. Am J Drug Alcohol Abuse 30:473-487.

Kendall-Tackett K (2002) The health effects of childhood abuse: four pathways by which abuse can influence health. Child Abuse Negl 26:715-729.

Langeland W, Hartgers C (1998) Child sexual and physical abuse and alcoholism: a review. J Studies Alcohol 59:336-348.

Leger D, Bayon V (2010) Societal costs of insomnia. Sleep Med Rev 14:379_ 389.

Li R, Wing Y, Ho S, Fong S (2002) Gender differences in insomnia - a study in the Hong Kong Chinese population. J Psychosomatic Res 53:601-609.

Mackenzie A, Funderburk FR, Allen RP (1999) Sleep, anxiety, and depression in abstinent and drinking alcoholics. Subst Use Misuse 34:347-361.

McCauley J, Kern DE, Kolodner K, Dill L, Schroeder AF, DeChant HK, Ryden J, Derogatis LR, Bass EB (1997) Clinical characteristics of women with a history of childhood abuse. J Am Med Assoc 277:1362-1368.

Mello N, Mendelson J (1970) Behavioral studies of sleep patterns in alcoholics during intoxication and withdrawal. J Pharmacol Exp Ther 175:94-112. 
Noll JG, Trickett PK, Susman EJ, Putnam FW (2006) Sleep disturbances and childhood sexual abuse. J Pediatric Psychol 31:469-480.

Pallesen S, Nordhus I, Nielsen G, Havik O, Kvale G, Johnsen B, Skjøtskift S (2001) Prevalence of insomnia in the adult Norwegian population. Sleep 24:771-779.

Peters EN, Fucito LM, Novosad C, Toll BA, O'Malley SS (2011) Effect of night smoking, sleep disturbance, and their co-occurrence on smoking outcomes. Psychol Addict Behav 25:312-319.

Pigeon WR, May PE, Perlis ML, Ward EA, Lu N, Talbot NL (2009) The effect of interpersonal psychotherapy for depression on insomnia symptoms in a cohort of women with sexual abuse histories. J Trauma Stress 22:634-638.

Pomerleau CS, Marks JL, Pomerleau OF (2000) Who gets what symptom? Effects of psychiatric cofactors and nicotine dependence on patterns of smoking withdrawal symptomatology. Nicotine Tobacco Res 2:275-280.

Regula J, Kulich KR, Stasiewicz J, Jasi ski B, Carlsson J, Wiklund I (2005) Stress in Polish patients with gastroesophageal reflux disease. Epidemiol $\mathrm{J}$ 59:75-85.

Selzer M (1971) The Michigan alcoholism screening test: the quest for a new diagnostic instrument. Am J Psychiatry 127:1653-1658.

Sheehan DV, Lecrubier Y, Sheehan KH, Amorim P, Janavs J, Weiller E, Hergueta T, Baker R, Dunbar GC (1998) The Mini-International Neuropsychiatric Interview (M.I.N.I.): the development and validation of a structured diagnostic psychiatric interview for DSM-IV and ICD-10. J Clin Psychiatry 59(Suppl 20):22-33.

Shinba T, Murashima YL, Yamamoto K (1994) Alcohol consumption and insomnia in a sample of Japanese alcoholics. Addiction 89:587-591.

Skoloda TE, Alterman A, Gottheil E (1979) Sleep quality reported by drinking and non-drinking alcoholics, in Addiction Research and Treatment: Converging Trends: Proceedings of the First Annual Coatesville-Jefferson Conference on Addiction (Gottheil EL, Altermnan AI, Druley KA, McLellan AT eds), pp 102-112. Pergamon Press, Elmsford, NY.

Smith G, Burnam M, Mosley C, Hollenberg J, Mancino M, Grimes W (2006) Reliability and validity of the substance abuse outcomes module. Psychiatric Serv 57:1452-1460.

Sobell L, Sobell M (1992) Timeline follow-back, in Measuring Alcohol Consumption: Psychosocial and Biochemical Methods (Litten RZ, Allen JP eds), pp 41-72. Humana Press, Totowa, NJ.
Soldatos CR, Allaert FA, Ohta T, Dikeos DG (2005) How do individuals sleep around the world? Results from a single-day survey in ten countries. Sleep Med 6:5-13.

Soldatos CR, Dikeos DG, Paparrigopoulos TJ (2000) Athens Insomnia Scale: validation of an instrument based on ICD-10 criteria. J Psychosomatic Res 48:555-560.

Soldatos CR, Kales JD, Scharf MB, Bixler EO, Kales A (1980) Cigarette smoking associated with sleep difficulty. Science 207:551-553.

Szczesniak P, Krecki R, Hoffman E, Orszulak-Michalak D (2008) Quality of life in patients with multivessel coronary heart disease. Contemporary Pharm 1:11-15.

Tabachnick BG, Fidell LS (2001) Using Multivariate Statistics. 4th ed. Allyn and Bacon, Boston, MA.

Taylor DJ, Lichstein KL, Durrence HH (2003) Insomnia as a health risk factor. Behav Sleep Med 1:227-247.

Teegen F (1999) Childhood sexual abuse and long-term sequelae, in Posttraumatic Stress Disorder: A Lifespan Developmental Perspective (Maercker A, Schutzwohl M, Solomon Z eds), pp 97-112. Hogrefe \& Huber, Seattle, WA.

Volk RJ, Cantor SB, Steinbauer JR, Cass AR (1997) Alcohol use disorders, consumption patterns, and health-related quality of life of primary care patients. Alcohol Clin Exp Res 21:899-905.

Ware JE, Kosinski M (2001) Interpreting SF-36 summary health measures: a response. Qual Life Res 10:405-413.

Ware JE, Sherbourne CD (1992) The MOS 36-item short-form health survey (SF-36): I. Conceptual framework and item selection. Med Care 30:473483.

Wetter DW, Young TB (1994) The relation between cigarette smoking and sleep disturbance. Prev Med 23:328-334.

Wong MM, Brower KJ, Fitzgerald HE, Zucker RA (2004) Sleep problems in early childhood and early onset of alcohol and other drug use in adolescence. Alcohol Clin Exp Res 28:578-587.

Wong MM, Brower KJ, Nigg JT, Zucker RA (2010) Childhood sleep problems, response inhibition, and alcohol and drug outcomes in adolescence and young adulthood. Alcohol Clin Exp Res 34:1033-1044.

Zhang B, Wing Y (2006) Sex differences in insomnia: a meta-analysis. Sleep 29:85-93. 